\title{
Identification of candidate genes and long non-coding RNAs associated with the effect of ATP5J in colorectal cancer
}

\author{
BINGJUN BAI ${ }^{1,2^{*}}$, BINBIN XIE $^{3 *}$, ZONGYOU PAN $^{4 *}$, LINA SHAN $^{1,2}$, JIANPEI ZHAO $^{5}$ and HONGBO ZHU ${ }^{1,2}$ \\ ${ }^{1}$ Department of Colorectal Surgery, Sir Run Run Shaw Hospital, School of Medicine, Zhejiang University; \\ ${ }^{2}$ Key Laboratory of Biotherapy of Zhejiang Province; ${ }^{3}$ Department of Medical Oncology, Sir Run Run Shaw Hospital, \\ School of Medicine, Zhejiang University; ${ }^{4}$ Department of Sports Medicine, School of Medicine, \\ Zhejiang University, Hangzhou, Zhejiang 310016; ${ }^{5}$ Department of Colorectal Surgery, \\ No. 2 Hospital of Ningbo, Ningbo, Zhejiang 315010, P.R. China
}

Received October 15, 2017; Accepted February 15, 2018

DOI: 10.3892/ijo.2018.4281

\begin{abstract}
The incidence and development of colorectal cancer (CRC) is a process with multiple gene interactions. We have previously demonstrated that ATP synthase-coupling factor 6, mitochondrial (ATP5J) is associated with CRC migration and 5-fluorouracil resistance; nevertheless, the exact molecular mechanism remains unclear. The following study uses microarray and bioinformatics methods to identify candidate genes and long non-coding RNAs (lncRNAs) in CRC cells (two pairs) with upregulated and downregulated ATP5J.Briefly, a total of 2,190 differentially expressed mRNAs (DEmRNAs) were sorted. Reverse transcription-quantitative polymerase chain reaction (RT-qPCR) was performed for 4 DEmRNAs to validate the results of microarray analysis. Functional annotation and pathway enrichment were analyzed for DEmRNAs using the Database for Annotation, Visualization and Integrated Discovery. Significantly enriched pathways included the regulation of gene expression and cell growth. The protein-protein interaction network was constructed, and AKT serine/threonine kinase 2 (AKT2) was considered as one of the hub genes. For further analysis, 51 DEmRNAs and 30 DElncRNAs were selected that were positively or negatively associated with the expression of ATP5J in the two cell pairs. X-inactive specific transcript (XIST), premature ovarian failure 1B $(P O F 1 B)$ and calmin $(C L M N)$ were identified in the DEmRNA-DElncRNA co-expression network. The expression of AKT2 and XIST in CRC cells was confirmed by RT-qPCR. To sum up, the candidate genes and IncRNAs,
\end{abstract}

Correspondence to: Dr Hongbo Zhu, Department of Colorectal Surgery, Sir Run Run Shaw Hospital, School of Medicine, Zhejiang University, 3 Qingchun East Road, Hangzhou, Zhejiang 310016, P.R. China

E-mail: 3101010@zju.edu.cn

*Contributed equally

Key words: colorectal cancer, ATP5J, microarray, long non-coding RNA, bioinformatics analysis as well as potential signaling pathways, which were identified using integrated bioinformatics analysis, could improve the understanding of molecular events involved in the function of ATP5J in CRC.

\section{Introduction}

For patients with colorectal cancer (CRC), the overall survival benefits of systemic treatments, including tumor resection, 5-fluorouracil (5-FU)-based chemotherapy and targeted therapies, have been firmly established $(1,2)$. Nevertheless, recurrence and metastasis caused by the drug resistance of tumor cells continue to obstruct significant therapeutic efficacy.

The development of CRC is a multi-gene, multi-step and multi-factor interactive process. The improvement of therapeutic efficacy significantly relies on improved studies of gene functions and mechanisms (3). It has been widely recognized that abnormal bioenergetics is one of the most common phenotypes of a number of tumor cells, including CRC (4). Changes in ATP synthetase are found in tumor cells and are considered to be associated with the energy metabolism and drug resistance within the tumor $(5,6)$.

ATP synthase-coupling factor 6 , mitochondrial (ATP5J) is a protein connecting $\mathrm{F} 0$ and $\mathrm{F} 1$, which are two components of ATP synthetase (7). ATP5J has been hypothesized to recover the activity of ATPase inhibited by oligomycin, as well as the exchange between ATP and inorganic phosphorus (8). Our previous study reported that ATP5J may be a tumor biomarker. Higher expression of ATP5J was found in CRC tissue compared with that in normal tissue, and in metastatic lymph nodes compared with that in primary tumors (9). In vitro experiments demonstrated that upregulation of ATP5J expression in DLD1 cells enhances cell migration and 5-FU resistance, while downregulation reverses this reaction. Investigation of ATP5J has been mainly focused on cardiovascular disease. Only a few studies have discussed the role of ATP5J in cancer, including renal carcinoma and hepatocellular carcinoma $(10,11)$. The molecular mechanism of ATP5J in CRC cells remains unknown.

Based on previous studies, the following study investigated differentially expressed mRNAs (DEmRNAs) and lncRNAs 
(DElncRNAs) using microarray and bioinformatics methods. The obtained results may provide valuable information on the function and mechanism of ATP5J in CRC, and a better understanding may be beneficial for the improvement of CRC management.

\section{Materials and methods}

Cell lines. In our previous study (9), the human colon cancer DLD1 cell line was used for cell transfection and stable colony selection. The expression of ATP5J was highly downregulated in clone 4, while it was highly upregulated in clone 2. Consequently, 4 DLD1 cells that were stably transfected with pcDNA3.1(+)/ATP5J plasmid (DLD1/A2), ATP5J short hairpin (sh)RNA plasmid (DLD1/SA4) and the corresponding control vectors (DLD1/C6 and DLD1/CA6), respectively, were selected and used in the present study.

RNA extraction and microarray. Total RNA was extracted from the 4 cell types by TRIzol reagent (Invitrogen; Thermo Fisher Scientific, Inc., Waltham, MA, USA) according to the manufacturer' s protocols. RNA quality was detected by an Agilent 2100 Bioanalyzer (Agilent Technologies, Inc., Santa Clara, CA, USA). The microarray analysis was performed by CapitalBio Corporation (Beijing, China). Total RNA was extracted from the cells and the cDNA that was then produced by reverse transcription served as the template to synthesize the fluorescently labeled (Cy5 or Cy3) cDNA using Klenow fragment polymerase. The labeled cDNA was hybridized on the lncRNA + mRNA Human Gene Expression Microarray (4x180K; Agilent Technologies, Inc.). The microarrays were washed three times with wash solution (0.2\% SDS, 2X SSC at $42^{\circ} \mathrm{C}$ for $120 \mathrm{sec}$ ) and scanned using an Agilent G2565CA Microarray Scanner (Agilent Technologies, Inc.), according to the manufacturer' s protocols. The raw data were analyzed and then normalized using percentile normalization. Only the mRNAs or lncRNAs with a fold-change $\geq 2.0$ and a P-value cutoff of $<0.05$ were considered as DEmRNAs or DElncRNAs.

Pathway enrichment analysis of DEmRNAs. The Database for Annotation, Visualization and Integrated Discovery (DAVID; https://david.ncifcrf.gov/) provides a comprehensive set of functional annotation tools for investigators to extract biological meaning from genes or proteins. The Kyoto Encyclopedia of Genes and Genomes (KEGG; http://www.kegg.jp/) is a database resource for functional interpretation and annotation of enriched pathways using large-scale gene datasets. The Gene Ontology (GO; http://www.geneontology.org/) database is commonly used to unify the representation of gene and gene product attributes. GO and KEGG pathway enrichment analyses were conducted for differentially expressed genes using DAVID. A P-value of $<0.05$ was selected as the cut-off criterion.

Protein-protein interaction (PPI) network construction. The Search Tool for the Retrieval of Interacting Genes (STRING; http://www.string-db.org/) database is an online database containing direct and indirect associations of proteins. DEmRNAs were mapped to STRING to assess the interactions.
Table I. Sequences of primers used for reverse transcriptionquantitative polymerase chain reaction.

\begin{tabular}{ll}
$\begin{array}{l}\text { mRNAs/ } \\
\text { lncRNAs }\end{array}$ & \multicolumn{1}{c}{ Primer sequences } \\
\hline ATP5J & F: TCAGCCGTCTCAGTCCATTT \\
& R: CCAAACATTTGCTTGAGCTT \\
CRTAM & F: CCAAATACCAGCTTCTTCATCA \\
& R: CTTCAAACCGGAAGGGTGCT \\
CD44 & F: AGTCACAGACCTGCCCAATGCCTTT \\
& R: TTTGCTCCACCTTCTTGACTCCCATG \\
CST1 & F: AGGAGACCATGGCCCAGTAT \\
& R: GCAGCGGACGTCTGTAGTAG \\
EEF1A2 & F: TCTCCAAGAATGGGCAGACG \\
& R: TTGACGATCTCGTCGTAGCG \\
& F: CTCTCCATTGGGTTCAC \\
AKT2 & R: GCGGCAGGTCTTAAGAGATGAG \\
& F: GCCACCATGAATGAGGTGAATA \\
RAPDH & R: CCTTGTACCCAATGAAGGAGC \\
& R: ACCACAGTCCATGCCATCAC \\
& RCACCACCCTGTTGCTGTA
\end{tabular}

ATP5J, ATP synthase-coupling factor 6, mitochondrial; CRTAM, cytotoxic and regulatory $\mathrm{T}$ cell molecule; CST1, cystatin SN; CD44, cluster of differentiation $44 ; E E F 1 A 2$, eukaryotic translation elongation factor $1 \alpha 2 ; A K T 2$, AKT serine/threonine kinase $2 ; X I S T, \mathrm{X}$-inactive specific transcript; lnCRNA, long non-coding RNA; F, forward; R, reverse.

Next, PPI networks were established using Cytoscape software (version 3.6.0; http://www.cytoscape.org/). The Molecular Complex Detection (MCODE) plug-in was utilized to filter the modules of the PPI network in Cytoscape. An MCODE score of $>5$ was the selection criteria. Hub genes were exported. Function and pathway enrichment analyses were performed for DEmRNAs in the modules. A P-value of $<0.05$ was considered statistically significant.

Reverse transcription-quantitative polymerase chain reaction (RT-qPCR). RNA was extracted as aforementioned. RNA was quantified using a NanoDrop 2000c spectrophotometer (Thermo Fisher Scientific, Inc.). cDNA was generated using an RNeasy Mini kit (Takara Bio, Inc., Otsu, Japan). qPCR analysis was performed with SYBR-Green Master mix (Takara Bio, Inc.) for lncRNA and mRNA detection. The qPCR was performed at $95^{\circ} \mathrm{C}$ for $2 \mathrm{~min}, 40$ cycles of $95^{\circ} \mathrm{C}$ for $5 \mathrm{sec}$ and $60^{\circ} \mathrm{C}$ for $30 \mathrm{sec}$, one cycle of $95^{\circ} \mathrm{C}$ for $5 \mathrm{sec}$, $60^{\circ} \mathrm{C}$ for $1 \mathrm{~min}$ and $95^{\circ} \mathrm{C}$ for $15 \mathrm{sec}$, and finally, $50^{\circ} \mathrm{C}$ for $30 \mathrm{sec}$. Relative expression was analyzed using the $2^{-\Delta \Delta \mathrm{Cq}}$ method (12). Human GAPDH was used as an endogenous reference gene. The sequences of the primers are shown in Table I.

Construction of DEmRNA-DElncRNA co-expression network. A DElncRNA-DEmRNA co-expression network was constructed to explore the association between the RNAs. Pearson's correlation coefficient (PCC) was calculated between 


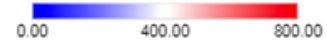

Comparison

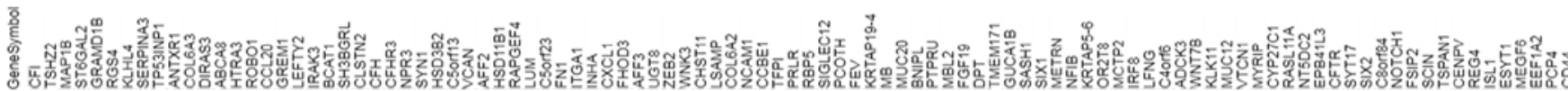
on

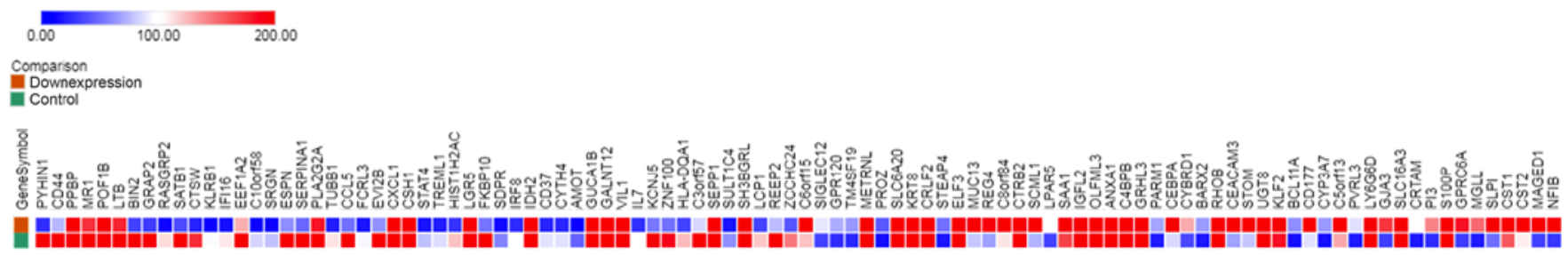

Figure 1. Heat map of the top 100 differentially expressed DEmRNAs in two pairs of cells. Red and blue represent high expression and low expression, respectively.
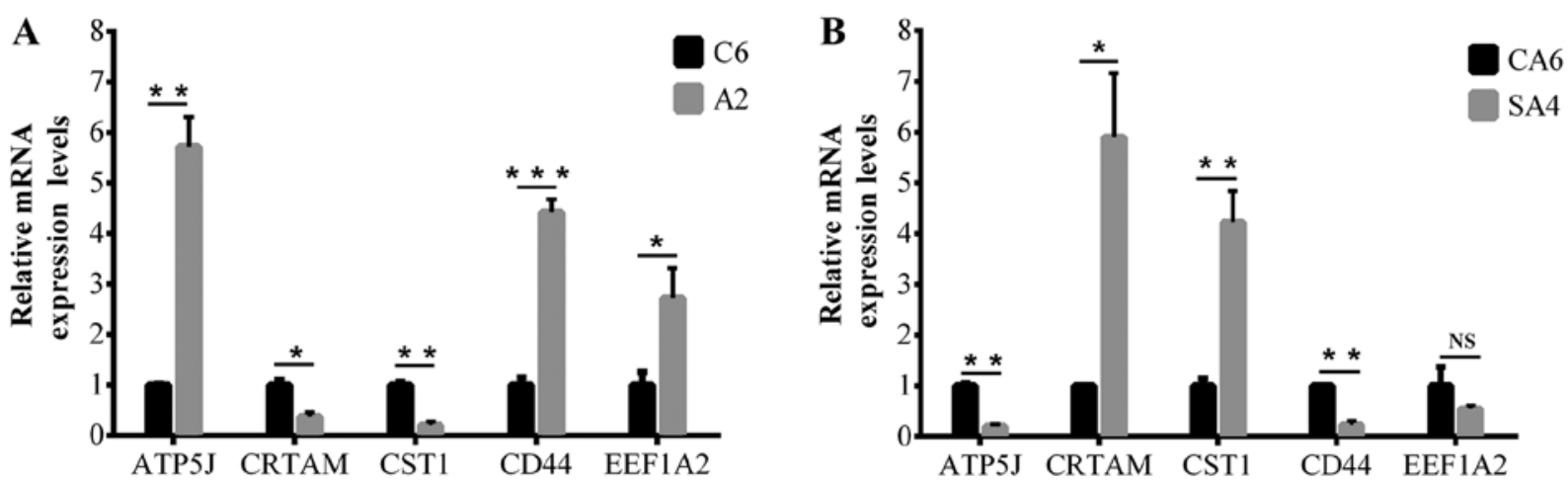

Figure 2. Reverse transcription-quantitative polymerase chain reaction results for ATP5J and four randomly selected mRNAs. (A) The expression level in DLD1/A2 and DLD1/C6; (B) The expression level in DLD1/SA4 and DLD1/CA6. ${ }^{*} \mathrm{P}<0.05,{ }^{* *} \mathrm{P}<0.01$ and ${ }^{* * *} \mathrm{P}<0.001$. NS, not significant; ATP5J, ATP synthase-coupling factor 6, mitochondrial; CRTAM, cytotoxic and regulatory T cell molecule; CST1, cystatin SN; CD44, cluster of differentiation 44; EEF1A2, eukaryotic translation elongation factor $1 \alpha 2$.

DElncRNAs and DEmRNAs according to corresponding expression levels. The criteria of $\mid \mathrm{PCCl} \geq 0.90$ was used to determine DElncRNA-DEmRNA pairs for network construction. The DElncRNA-DEmRNA network was constructed using Cytoscape 3.5.1 (http://cytoscape.org/).

Statistical analysis. The results of the qPCR were presented using Graph Pad Prism (version 6.0; GraphPad Software, Inc., La Jolla, CA, USA). Data are presented as the mean \pm standard error of the mean. The independent samples t-test was performed for data comparison. $\mathrm{P}<0.05$ was considered to indicate a statistically significant difference.

\section{Results}

Identification of DEmRNAs. According to microarray analysis, 32,206 mRNAs were detected in two pairs of cells, including 2,190 DEmRNAs (503 upregulated and 1,687 downregulated), which were significantly differentially expressed with a fold-change of $\geq 2.0$. In addition, the DEmRNAs were divided into two sets: Set A contained all DEmRNAs from cell pair DLD1/A2 vs. DLD1/C6, and set B contained all DEmRNAs from DLD1/SA4 vs. DLD1/CA6. Heat map representation showed the top 100 differentially DEmRNAs (Fig. 1).

$R T$-qPCR validation of DEmRNAs. To validate the results of microarray analysis, RT-qPCR was performed in ATP5J and four randomly selected mRNAs. As shown in Fig. 2, cytotoxic and regulatory $\mathrm{T}$ cell molecule and cystatin $\mathrm{SN}$ were downregulated in DLD1/A2 vs. DLD1/C6 and upregulated in DLD1/SA4 vs. DLD1/CA6, while opposite results were obtained for ATP5J, cluster of differentiation 44 and eukaryotic translation elongation factor $1 \alpha 2$. The PCR results were basically concurrent with the microarray data.

Functional and pathway enrichment analysis. In order to investigate potential gene and gene products, GO functional and KEGG pathway enrichment analyses were performed for sets A and B, respectively. The DEmRNAs from set A were mainly enriched in biological processes associated with 'positive regulation of gene expression', 'cell-cell signaling' and 'regulation of cell growth'. The 10 most significantly enriched GO terms for DEmRNAs from sets A and B are shown in Fig. 3A and $\mathrm{C}$, while the 10 most significantly 

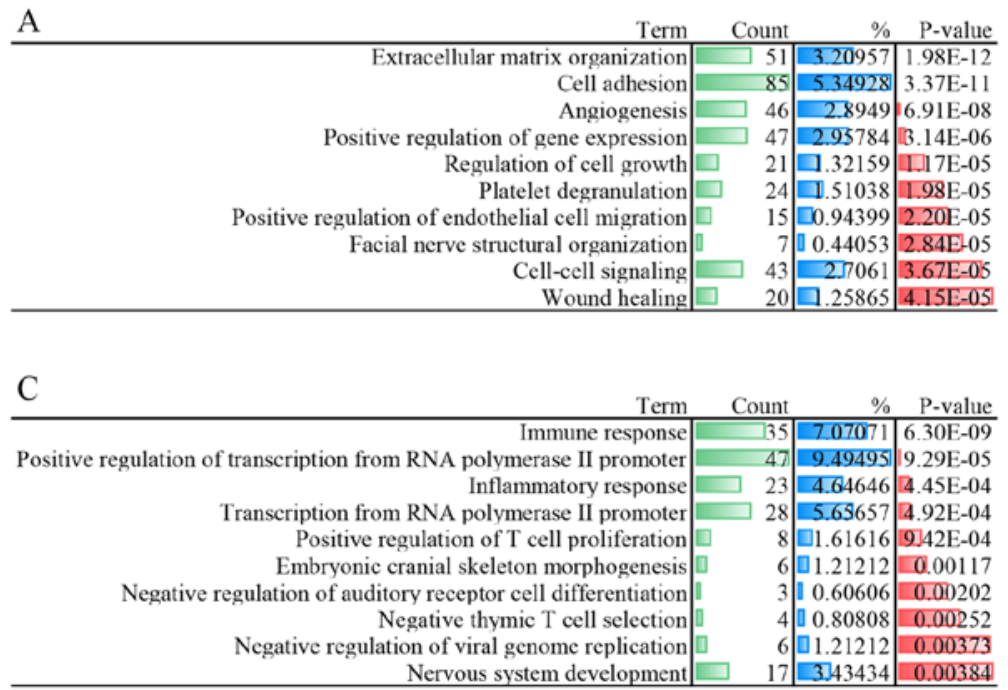
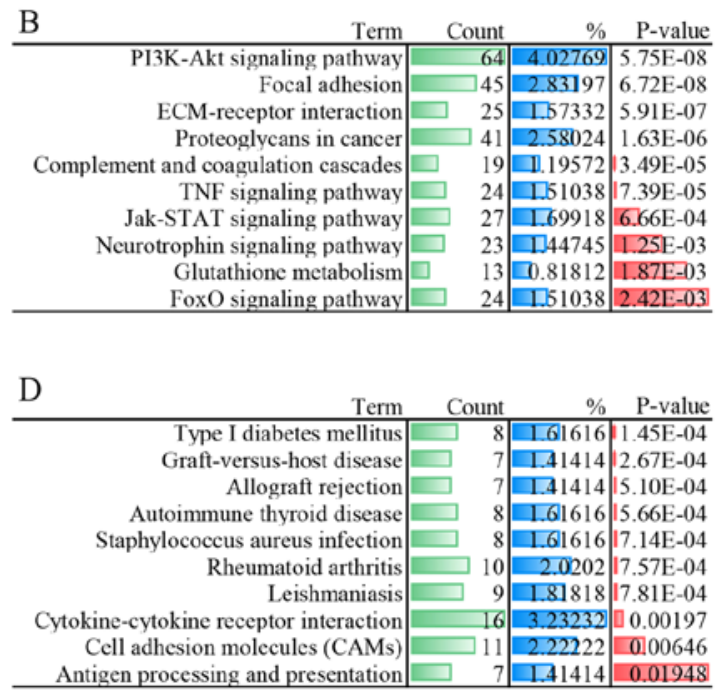

Figure 3. Gene Ontology functional and KEGG pathway enrichment analysis. The top 10 terms were selected according to P-value. (A) Biological process of set A. (B) Enriched KEGG pathways of set A. (C) Biological process of set B. (D) Enriched KEGG pathways of set B. KEGG, Kyoto Encyclopedia of Genes and Genomes.

enriched KEGG pathways in sets $\mathrm{A}$ and $\mathrm{B}$ are presented in Fig. 3B and D.

PPI network analysis. By submitting all DEmRNAs from sets A and B into STRING, the PPI network was obtained (data not shown). Next, the MCODE plug-in was used to analyze the results of STRING in Cytoscape software. The 3 most significant modules were acquired; the functions were mainly associated with 'cytokine-cytokine receptor interaction', 'ubiquitin-mediated proteolysis' and 'cell adhesion molecules (CAMs)' (Fig. 4). Moreover, a total of 20 genes were considered as hub genes (Table II), among which AKT serine/threonine kinase 2 (AKT2) obtained the highest value for neighborhood connectivity, outdegree and closeness centrality. This meant that AKT2 was the most important gene within the PPI network and represented the closest connection with other genes in the module (13).

Co-expression network of DEmRNA-DElncRNA. Based on microarray analysis, 39,304 lncRNAs were detected. A total of 3,002 DElncRNAs (2,319 in DLD1/A2 vs. DLD1/C6; 683 in DLD1/SA4 vs. DLD1/CA6) were significantly differentially expressed with a fold-change of $\geq 2.0$. For further analysis, DElncRNAs upregulated in DLD1/A2 vs. DLD1/C6 and downregulated in DLD1/SA4 vs. DLD1/CA6 were selected, which meant that the expression of DElncRNAs was positively associated with the expression of ATP5J in the two cell pairs. The DElncRNAs that were negatively associated with ATP5J in the two cell pairs were also included. Certain DEmRNAs were selected according to the aforementioned criteria. Therefore, 51 DEmRNAs and 30 DElncRNAs were identified and listed in Tables III and IV.

To further investigate the potential association between DEmRNAs and DElncRNAs, PCC was calculated according to the expression levels of 51 DEmRNAs and 30 DElncRNAs. Using $\mid P C C l \geq 0.90,343$ DEmRNA/DElncRNA pairs were used to construct the co-expression network, including 239 pairs exhibiting positive correlation. The network is shown
Table II. Hub genes from Molecular Complex Detection analysis.

\begin{tabular}{lccc}
\hline Gene symbol & $\begin{array}{c}\text { Neighborhood } \\
\text { connectivity }\end{array}$ & Outdegree & $\begin{array}{c}\text { Closeness } \\
\text { centrality }\end{array}$ \\
\hline MTNR1A & 42.20 & 12 & $3.23 \times 10^{-1}$ \\
SPSB1 & 30.65 & 8 & $2.93 \times 10^{-1}$ \\
CCKBR & 47.25 & 8 & $2.94 \times 10^{-1}$ \\
AKT2 & 57.50 & 34 & $3.54 \times 10^{-1}$ \\
GPX3 & 16.18 & 15 & $2.62 \times 10^{-1}$ \\
PTPN6 & 41.71 & 31 & $3.38 \times 10^{-1}$ \\
CLIC3 & 17.20 & 5 & $2.28 \times 10^{-1}$ \\
TNFRSF19 & 10.00 & 6 & $2.56 \times 10^{-1}$ \\
IFT80 & 45.83 & 2 & $2.03 \times 10^{-1}$ \\
AMHR2 & 34.69 & 1 & $2.93 \times 10^{-1}$ \\
MAMLD1 & 41.00 & 8 & $2.96 \times 10^{-1}$ \\
DHRSX & 30.63 & 3 & $1.93 \times 10^{-1}$ \\
SLC47A1 & 5.00 & 1 & 1.00 \\
SLC30A4 & 3.75 & 0 & 0.00 \\
CYBRD1 & 19.33 & 1 & 1.00 \\
INTS6 & 14.67 & 0 & 0.00 \\
CLDN7 & 9.50 & 4 & $2.04 \times 10^{-1}$ \\
TNFSF12 & 56.67 & 9 & $2.93 \times 10^{-1}$ \\
DGKA & 21.50 & 3 & $3.13 \times 10^{-1}$ \\
HOMER2 & 27.13 & 2 & $2.49 \times 10^{-1}$
\end{tabular}

${ }^{\mathrm{a}}$ Gene showing the closest connection with other genes.

in Fig. 5. The pair of premature ovarian failure 1B (POF1B) and ENSG00000236654 presented the most significant positive correlation coefficiency, while the most marked negative correlation coefficiency was found in the pair of glycogen phosphorylase L and ENSG00000170011. One DElncRNA may target multiple DEmRNAs; for example, XIST was 
A

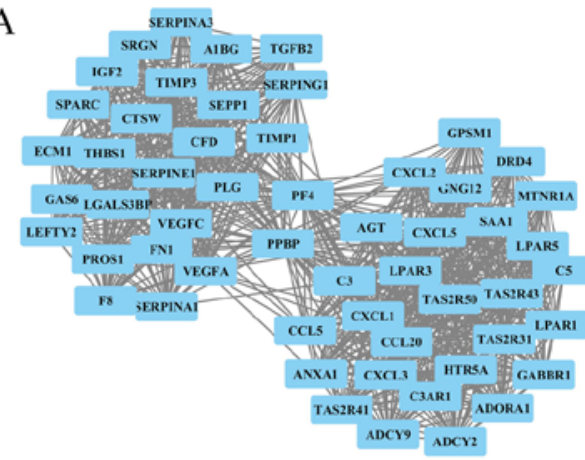

B

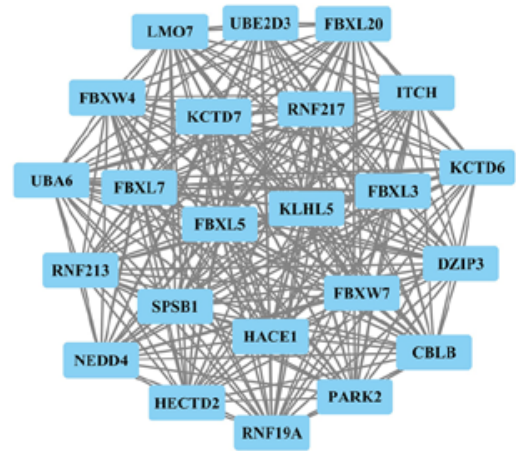

C

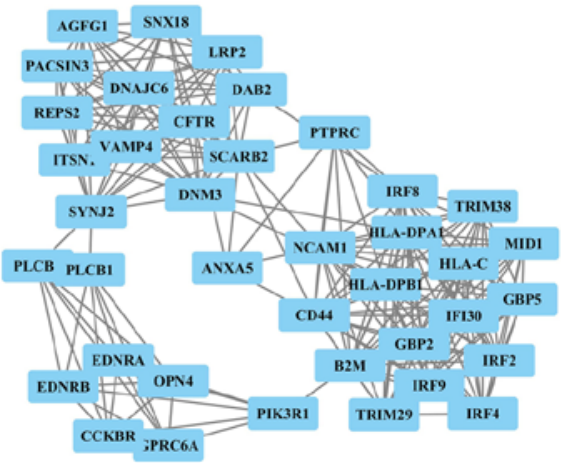

D

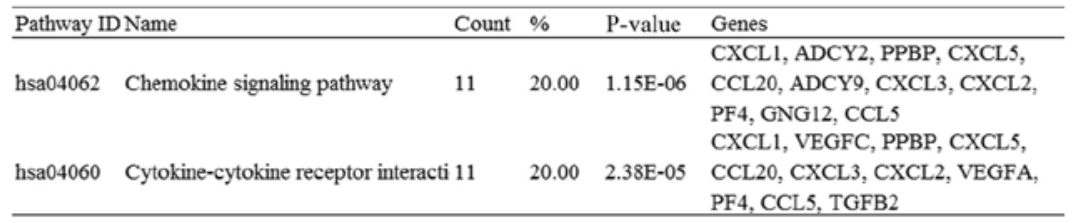

E

\begin{tabular}{|c|c|c|c|c|c|}
\hline \multicolumn{2}{|c|}{ Pathway ID Name } & \multirow{2}{*}{$\begin{array}{l}\text { Count } \\
7\end{array}$} & \multirow{2}{*}{$\%$} & \multirow{2}{*}{$\begin{array}{l}\text { P-value } \\
2.35 \mathrm{E}-09\end{array}$} & \multirow{2}{*}{$\begin{array}{l}\text { Genes } \\
\text { UBE2D3, FBXW7, CBLB, NEDD4, } \\
\text { UBA6, ITCH, PARK2 }\end{array}$} \\
\hline hsa04120 & Ubiquitin mediated proteolysis & & & & \\
\hline hsa04144 & Endocytosis & 3 & 13.04 & $2.43 \mathrm{E}-02$ & CBLB, NEDD4, ITCH \\
\hline
\end{tabular}

F

\begin{tabular}{|c|c|c|c|c|c|}
\hline \multicolumn{2}{|c|}{ Pathway ID Name } & \multirow{2}{*}{ Count } & \multirow{2}{*}{$\%$} & \multirow{2}{*}{$\begin{array}{l}\text { P-value } \\
2.62 \mathrm{E}-03\end{array}$} & \multirow{2}{*}{$\begin{array}{l}\text { Genes } \\
\text { NCAM1, PTPRC, HLA-C, HLA- } \\
\text { DPA1, HLA-DPB1 }\end{array}$} \\
\hline hsa04514 & Cell adhesion molecules (CAMs) & & & & \\
\hline hsa04070 & Phosphatidylinositol signaling syster 4 & & 10.26 & $4.25 \mathrm{E}-03$ & PLCB4, SYNJ2, PLCB1, PIK3R1 \\
\hline hsa04020 & Calcium signaling pathway & 5 & 12.82 & $7.33 \mathrm{E}-03$ & $\begin{array}{l}\text { EDNRA, EDNRB, PLCB } 4, \text { CCKBR, } \\
\text { PLCB1 }\end{array}$ \\
\hline
\end{tabular}

Figure 4. Top three modules of the protein-protein interaction network. (A) Module 1, (B) module 2, (C) module 3 and their genes, combined with enriched pathways, are shown in excel format in (D, E and F).

associated with $P O F 1 B$, chorionic somatomammotropin hormone 1 (CSH1) and calmin (CLMN), leading to multiple signaling pathways or mechanisms.

RT-qPCR validation of hub genes and DElncRNA. RT-qPCR was used to validate $A K T 2$ and XIST. As shown in Fig. 6, AKT2 and XIST were downregulated in DLD1/A2 vs. DLD1/C6 $(\mathrm{P}<0.01)$ and upregulated in DLD1/SA4 vs. DLD1/CA6 $(\mathrm{P}<0.05)$. In general, the validation results were consistent with the microarray data.

\section{Discussion}

ATP5J has been associated with several cancer types, including diffuse large B-cell lymphoma renal carcinoma and hepatocellular carcinoma $(10,11,14)$. Furthermore, our previous study confirmed that ATP5J is associated with CRC cell migration and 5-fluorouracil resistance. The present study further investigated the molecular mechanism and potential key genes interacting with ATP5J in CRC via microarray and bioinformatics analyses.
The mRNAs from set A, which were differentially expressed in the upregulation of ATP5J, were mainly enriched in the GO terms of 'extracellular matrix organization', 'cell adhesion', 'positive regulation of gene expression' and 'regulation of cell growth'. These biological processes are essential for tumor cell survival and growth. Emerging data have confirmed the crucial role of the extracellular matrix in tumor metastasis $(15,16)$. Changes in the content of the extracellular matrix may influence tumor cell properties, including proliferation and motility (17). Moreover, the primary enriched GO terms of set B contained enzyme regulation ('regulation of transcription from RNA polymerase II promoter') and biological responses ('immune response' and 'inflammatory response'). It is widely accepted that a causal association exists between inflammation, immunity and cancer (18). Human immunity responses, such as immune surveillance, are part of an innate and efficient antineoplastic system. Therefore, it could be concluded that the upregulation of ATP5J may affect cell migration and 5-FU sensitivity by influencing the biological processes of the intracellular and extracellular environment, thus promoting cell growth; 
Table III. Selected differentially expressed mRNAs for co-expression network construction $(\mathrm{n}=51)$.

\begin{tabular}{|c|c|c|c|}
\hline Ensemble ID & Gene symbol & $\log _{2} \mathrm{FC}$ (A2 vs. C6) & $\log _{2} \mathrm{FC}$ (SA4 vs. CA6) \\
\hline \multicolumn{4}{|l|}{ Upregulation } \\
\hline ENST00000463422 & АТОН8 & 2.3664742 & -1.5749975 \\
\hline ENST00000308108 & CCNE2 & 1.0017490 & -1.0507374 \\
\hline ENST00000278385 & $C D 44$ & 3.8303200 & -3.9564400 \\
\hline ENST00000298912 & $C L M N$ & 1.2790971 & -1.2013025 \\
\hline ENST00000329882 & CSH1 & 1.2013025 & -2.2145548 \\
\hline ENST00000249749 & DLL4 & 1.4630564 & -1.3910798 \\
\hline ENST00000217182 & EEF1A2 & 3.5102847 & -2.7776937 \\
\hline ENST00000371021 & FRAT1 & 1.1014051 & -1.1662812 \\
\hline ENST00000435292 & $G U C A 1 B$ & 1.7285424 & -1.9108304 \\
\hline ENST00000377791 & HIST1H2AC & 1.0301589 & -2.0924091 \\
\hline ENST00000330062 & IDH2 & 1.3220385 & -2.0191135 \\
\hline ENST00000518982 & $I L 7$ & 1.3051137 & -1.8685079 \\
\hline ENST00000268638 & $I R F 8$ & 1.8297353 & -2.0302430 \\
\hline ENST00000378111 & KCNAB2 & 1.1377705 & -1.4946451 \\
\hline ENST00000394683 & KIAA1199 & 1.0515032 & -1.0550995 \\
\hline ENST00000420981 & $M G C 39372$ & 1.2202988 & -1.6457891 \\
\hline ENST00000396217 & MYRIP & 1.9900737 & -1.2685270 \\
\hline ENST00000277541 & NOTCHI & 2.4109862 & -1.5415106 \\
\hline ENST00000276124 & POF $1 B$ & 1.1683197 & -3.3742200 \\
\hline ENST00000313755 & PRODH & 1.1842122 & -1.4703089 \\
\hline ENST00000341901 & $S B K 1$ & 1.1663734 & -1.3532438 \\
\hline ENST00000247182 & SIX1 & 1.7563076 & -1.2851509 \\
\hline ENST00000393062 & SPIRE2 & 1.3516588 & -1.1577921 \\
\hline ENST00000328526 & $X K R X$ & 1.1844625 & -1.1355352 \\
\hline \multicolumn{4}{|l|}{ Downregulation } \\
\hline ENST00000376726 & ACSS1 & -1.4264563 & 1.0032806 \\
\hline ENST00000374111 & C10orf53 & -3.0494199 & 2.7619016 \\
\hline ENST00000397899 & C2orf55 & -1.0406728 & 1.8891068 \\
\hline ENST00000492730 & $C 4 B P B$ & -1.8177981 & 1.7743891 \\
\hline ENST00000509979 & C5orf13 & -4.4611087 & 2.1796174 \\
\hline ENST00000503763 & $C R H B P$ & -2.4869404 & 1.2709899 \\
\hline ENST00000227348 & CRTAM & -2.1076543 & 2.4206290 \\
\hline ENST00000450719 & CSHL1 & -1.2476245 & 1.3876114 \\
\hline ENST00000398402 & CST1 & -1.7663736 & 3.7468631 \\
\hline ENST00000304725 & CST2 & -3.1294575 & 4.1763420 \\
\hline ENST00000292414 & CYP3A7 & -1.5009923 & 2.0561150 \\
\hline ENST00000317811 & $F J X 1$ & -1.1050119 & 1.2775639 \\
\hline ENST00000310357 & GPRC6A & -1.3399930 & 2.4899235 \\
\hline ENST00000393584 & GSTA5 & -1.7383062 & 1.0165440 \\
\hline ENST00000309446 & $K L F 7$ & -1.5819120 & 1.1883107 \\
\hline ENST00000375827 & LY6G6D & -1.1885548 & 2.3284416 \\
\hline ENST00000427231 & $N E B$ & -2.4703722 & 1.1157570 \\
\hline ENST00000319792 & PVRL3 & -4.1147676 & 2.2300897 \\
\hline ENST00000216392 & $P Y G L$ & -3.4774067 & 1.2769729 \\
\hline ENST00000405158 & $S A A 1$ & -3.2550786 & 1.6204370 \\
\hline ENST00000414546 & $S A A 2$ & -1.4848727 & 1.1160498 \\
\hline ENST00000051659 & SCML1 & -2.6339436 & 1.5956994 \\
\hline ENST00000486749 & $S L C 2 A 3$ & -2.8174708 & 1.1550741 \\
\hline ENST00000358525 & SLC6A20 & -1.2244166 & 1.4633511 \\
\hline ENST00000338380 & $S L P I$ & -1.9716522 & 3.2052798 \\
\hline ENST00000394511 & $U G T 8$ & -4.2315920 & 1.8874178 \\
\hline
\end{tabular}

FC, fold-change. 


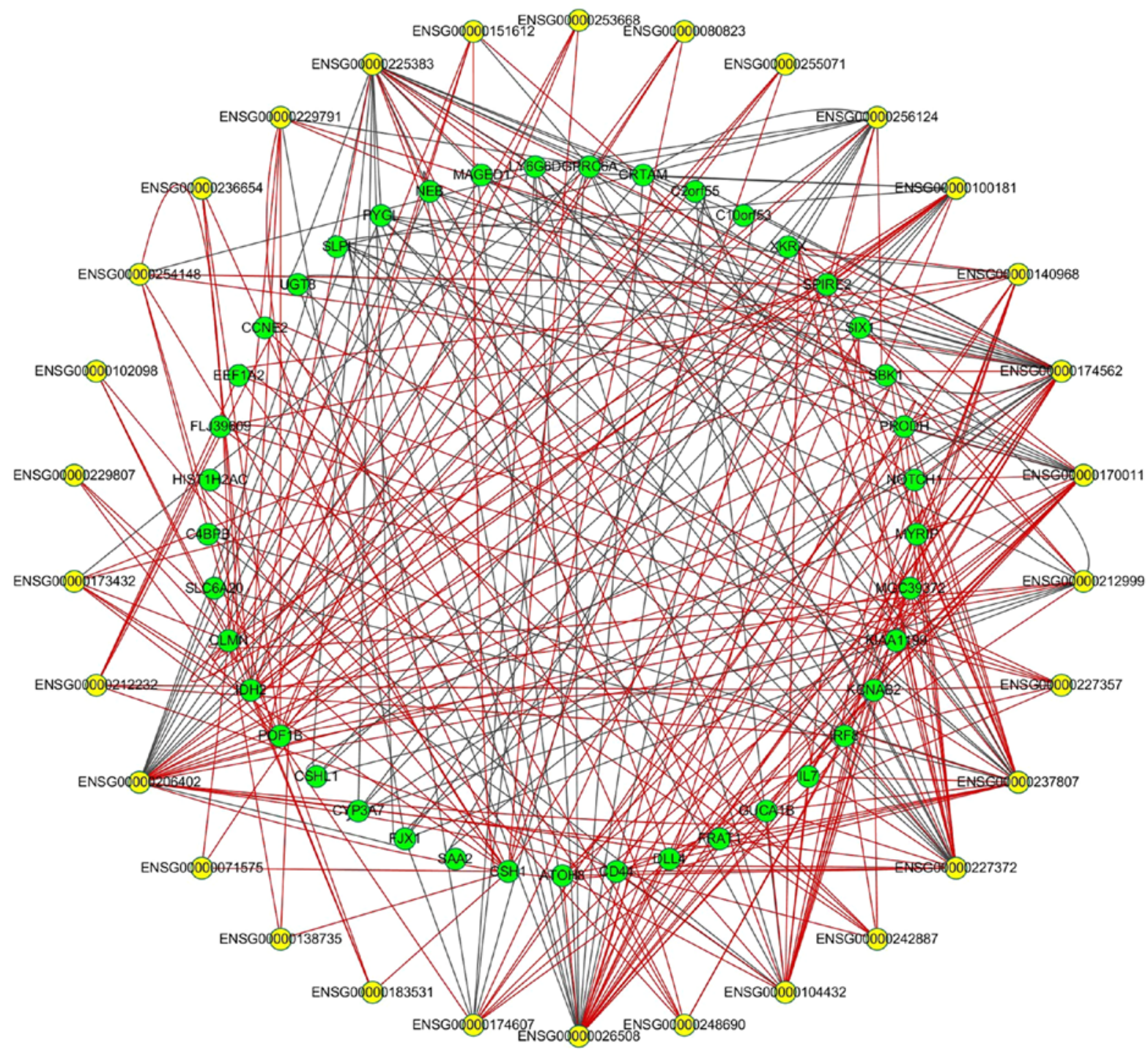

Figure 5. DElncRNA-DEmRNA co-expression network constructed based on the correlation analysis. Yellow nodes represent DElncRNAs and green nodes represent the target DEmRNAs. Red lines represent a positive association and black lines represent a negative association. DElncRNA, differentially-expressed long non-coding RNA.
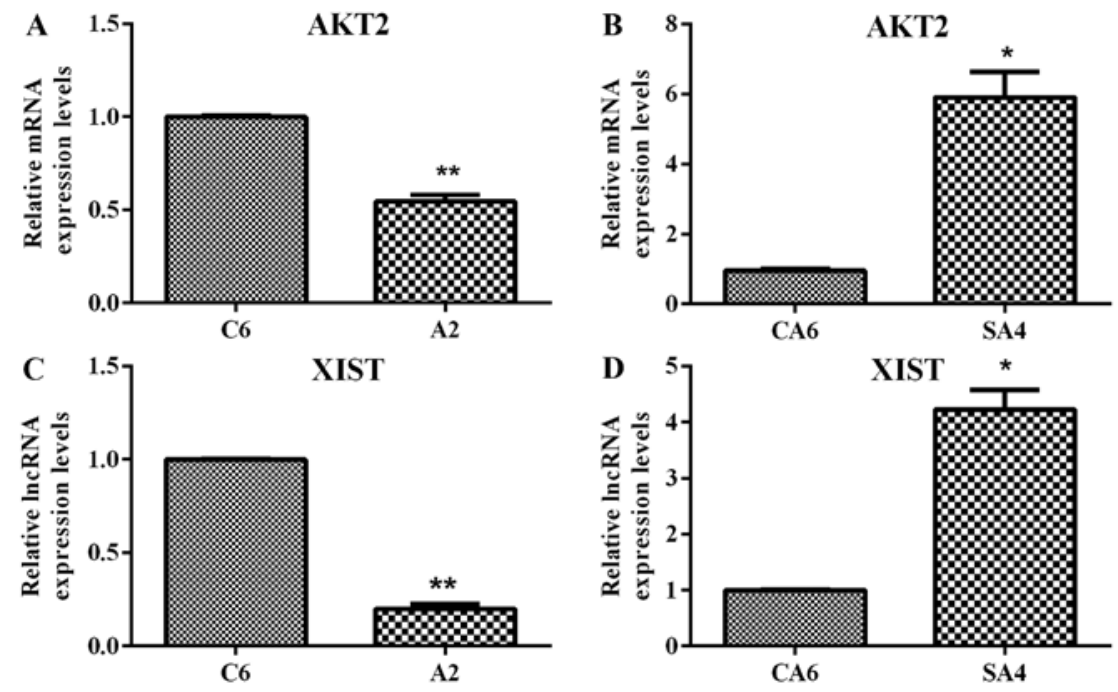

Figure 6. Reverse transcription-quantitative polymerase chain reaction validation of $A K T 2$ and XIST. (A) The expression level of AKT2 in DLD1/A2 and DLD1/C6. (B) The expression level of $A K T$ in DLD1/SA4 and DLD1/CA6. (C) The expression level of XIST in DLD1/A2 and DLD1/C6. (D) The expression level of XIST in DLD1/SA4 and DLD1/CA6. "P<0.05 and ${ }^{* *} \mathrm{P}<0.01$. lncRNA, long non-coding RNA; AKT2, AKT serine/threonine kinase 2; XIST, X-inactive specific transcript. 
Table IV. Selected differentially expressed long non-coding RNAs for co-expression network construction $(\mathrm{n}=30)$.

\begin{tabular}{|c|c|c|c|}
\hline Ensemble gene ID & Gene symbol & $\log 2 \mathrm{FC}$ (A2 vs. C6) & $\log 2 \mathrm{FC}$ (SA4 vs. CA6) \\
\hline \multicolumn{4}{|l|}{ Upregulation } \\
\hline ENSG00000026508 & $C D 44$ & 5.7112436 & -4.6520596 \\
\hline ENSG00000248690 & $H A S 2-A S 1$ & 1.5613460 & -1.3604736 \\
\hline ENSG00000104432 & $I L 7$ & 1.2327710 & -2.0651786 \\
\hline ENSG00000242887 & IGHJ3 & 1.1636353 & -1.1661916 \\
\hline ENSG00000227372 & TP73-AS1 & 1.1705691 & -1.1062335 \\
\hline ENSG00000237807 & RP11-400K9.4 & 1.0098063 & -1.4481292 \\
\hline ENSG00000227357 & $H L A-D R B 4$ & 1.0012778 & -1.1985005 \\
\hline ENSG00000212999 & AC117834.1 & 1.2642217 & -1.2010332 \\
\hline ENSG00000170011 & MYRIP & 1.9657596 & -1.1791620 \\
\hline ENSG00000174562 & KLK15 & 1.7544044 & -1.0959578 \\
\hline ENSG00000140968 & $I R F 8$ & 2.0519360 & -1.8488283 \\
\hline \multicolumn{4}{|l|}{ Downregulation } \\
\hline ENSG00000100181 & TPTEP1 & -1.1547785 & 1.0562963 \\
\hline ENSG00000256124 & $R P 11-84 E 24.3$ & -1.0987811 & 1.4957023 \\
\hline ENSG00000255071 & $S A A 2-S A A 4$ & -2.9303002 & 1.6360683 \\
\hline ENSG00000080823 & $M O K$ & -1.2133837 & 1.0177355 \\
\hline ENSG00000253668 & RP11-463C14.1 & -3.7030535 & 2.1768303 \\
\hline ENSG00000151612 & ZNF827 & -1.9309711 & 1.2170410 \\
\hline ENSG00000225383 & SFTAIP & -1.1355629 & 1.3586316 \\
\hline ENSG00000229791 & LINC00420 & -1.6585293 & 1.3905687 \\
\hline ENSG00000236654 & AC079780.3 & -4.6469680 & 1.8889217 \\
\hline ENSG00000254148 & RP11-84E24.2 & -2.1362443 & 1.6749586 \\
\hline ENSG00000102098 & $S C M L 2$ & -1.3423405 & 1.0036592 \\
\hline ENSG00000229807 & XIST & -2.4771900 & 1.0491910 \\
\hline ENSG00000173432 & $S A A l$ & -1.9162921 & 1.2718056 \\
\hline ENSG00000212232 & SNORD17 & -1.2717161 & 1.0156708 \\
\hline ENSG00000206402 & LY6G6D & -1.5264271 & 2.9315690 \\
\hline ENSG00000071575 & TRIB2 & -1.3852354 & 1.0461107 \\
\hline ENSG00000138735 & PDE5A & -1.5525875 & 1.1479750 \\
\hline ENSG00000183531 & Z98256.1 & -2.9538560 & 2.0470590 \\
\hline ENSG00000174607 & $U G T 8$ & -3.3867934 & 2.0097785 \\
\hline
\end{tabular}

FC, fold-change.

its downregulation may operate by regulating immune and inflammatory responses. The KEGG pathways of set A were enriched primarily in tumor-related processes, including the phosphoinositide 3-kinase-protein kinase B signaling pathway, the tumor necrosis factor (TNF) signaling pathway and the Janus kinase-signal transducer and activator of transcription signaling pathway $(19,20)$.

According to PPI network analysis, several biomarkers were identified. Some of these genes are associated with CRC biological behavior, including AKT2, TNF receptor superfamily member 19 , claudin 7 , cholecystokinin B receptor, glutathione peroxidase 3 and homer scaffolding protein 2 . $A K T 2$, one of the most significantly meaningful genes, has been shown to be essential in several cellular pathways involved in cell proliferation, metastasis and drug resistance (21). Overexpression of AKT2 has been detected in breast, ovarian and colon cancer $(22,23)$. Previous studies have investigated the role of AKT2 in CRC via the construction of cell and animal models. The results showed that AKT2 deficiency may decrease the metastatic ability of CRC cells in mice (24). In addition, an shRNA-mediated AKT2-knockdown system has been shown to decrease cell survival and proliferation in primary tumors (25). Furthermore, low AKT2 expression in HCT116 cells has been shown to be correlated with increased chemosensitivity to paclitaxel (26). Taken together, the regulation of AKT2 may be a critical process for ATP5J exerting an effect on CRC cell biological functions.

Numerous studies have confirmed the association between IncRNAs and tumors. Previous studies have indicated that lncRNAs could have an important regulatory role in gene expression and are associated with cancer development (27). In the present study, microarray analysis was performed for lncRNAs. From the lncRNA expression profiles, DElncRNAs meeting the criteria were selected for further analysis. According to the results, TP73-AS1, HAS2-AS1, SFTA1P and $X I S T$ were highlighted and have been previously associated 
with the development and progression of certain cancer types, including hepatocellular carcinoma and oral squamous cell carcinoma (28-30). Among them, XIST was the only lncRNA that has been previously investigated in CRC (31). XIST, located in the $\mathrm{X}$ chromosome, was one of the first lncRNAs to be found that was involved in the progression of cancer, including non-small cell lung cancer and human glioblastoma $(32,33)$. Certain studies have found the mechanism of XIST in several cancer types; for example, the lncRNA XIST has been shown to promote the malignancy of esophageal squamous cell carcinoma through the modulation of miR-101/ enhancer of zeste homolog 2 (34). In addition, by regulating miR-186-5p, XIST controls cell proliferation and invasion in non-small cell lung cancer (35). Moreover, significantly high XIST expression has been found in CRC tumor tissues compared with that in paired adjacent normal tissues (36). High-level expression of XIST could predict poor disease-free survival times in CRC patients. Moreover, XIST is considered to be an oncogene that could promote cell proliferation through the miR-132-3p/mitogen-activated protein kinase 1 axis (31)

To further elucidate the potential functions of lncRNAs and find out their target genes in the cell pairs of the present study, a co-expression network of DEmRNA/DElncRNA was constructed. XIST was associated with 3 mRNAs (POFIB, $C S H 1$ and $C L M N)$. The gene encoding POF1B was critical for ovarian function (37). However, its impact was also confirmed in the regulation of cell adhesion in human intestinal cell lines (38). CLMN has been shown to regulate the cell cycle in a report involving neuroblastoma cells (39). Therefore, we hypothesized that the co-repression of XIST and POF1B and CLMN was involved in the molecular mechanisms of ATP5J in CRC cells, which will be confirmed by further cell line experiments in future studies.

In conclusion, the present study determined the expression profiles of mRNAs and lncRNAs in CRC cells overexpressing/ underexpressing ATP5J. According to bioinformatics analyses, $A K T 2$ and XIST expression was identified as a potential biomarker participating in the effect of ATP5J in CRC, which in turn was associated with cell migration and 5-FU resistance. Further research is necessary to confirm this hypothesis.

\section{Acknowledgements}

This study represents partial fulfillment of the requirements for a Master degree for Dr Bingjun Bai.

\section{Funding}

This study was supported by the National Natural Science Foundation of China (grant no. 81272681) and the Medical and Health Science and Technology Foundation of Zhejiang Province (grant no. 2017 KY593).

\section{Availability of data and materials}

The datasets analyzed during the current study are not publicly available as they will be used in our further studies, but they are available from the corresponding author on reasonable request.

\section{Authors' contributions}

Guarantor of integrity of entire study: BJB, BBX, ZYP and HBZ. Study concepts: BJB, BBX, ZYP and HBZ. Study design: BJB, BBX, ZYP and HBZ. Data acquisition: LNS and ZJP. Data analysis/interpretation: LNS and ZJP. Statistical analysis: BJB, BBX, ZYP and HBZ. Manuscript preparation: LNS and ZJP. Manuscript revision/review: BJB, BBX, ZYP and HBZ. Manuscript final version approval: BJB, BBX, ZYP and HBZ.

\section{Ethics approval and consent to participate}

Not applicable.

\section{Consent for publication}

Not applicable.

\section{Conflicts of interest}

All authors listed in the manuscript agree on the content of the and this submission. The authors declare no conflict of interest.

\section{References}

1. Chau I and Cunningham D: Chemotherapy in colorectal cancer: New options and new challenges. Br Med Bull 64: 159-180, 2002.

2. Shao YC, Chang YY, Lin JK, Lin CC, Wang HS, Yang SH, Jiang JK, Lan YT, Lin TC, Li AF, et al: Neoadjuvant chemotherapy can improve outcome of colorectal cancer patients with unresectable metastasis. Int J Colorectal Dis 28: 1359-1365, 2013.

3. Liu HY and Zhang CJ: Identification of differentially expressed genes and their upstream regulators in colorectal cancer. Cancer Gene Ther 24: 244-250, 2017.

4. Pedersen PL: Bioenergetics of cancer cells - A brief orientation to this minireview series. J Bioenerg Biomembr 29: 301-302, 1997.

5. Cuezva JM, Krajewska M, de Heredia ML, Krajewski S, Santamaría G, Kim H, Zapata JM, Marusawa H, Chamorro M and Reed JC: The bioenergetic signature of cancer: A marker of tumor progression. Cancer Res 62: 6674-6681, 2002.

6. Shin YK, Yoo BC, Chang HJ, Jeon E, Hong SH, Jung MS, Lim SJ and Park JG: Down-regulation of mitochondrial F1F0-ATP synthase in human colon cancer cells with induced 5-fluorouracil resistance. Cancer Res 65: 3162-3170, 2005.

7. Collinson IR, van Raaij MJ, Runswick MJ, Fearnley IM, Skehel JM, Orriss GL, Miroux B and Walker JE: ATP synthase from bovine heart mitochondria. In vitro assembly of a stalk complex in the presence of F1-ATPase and in its absence. J Mol Biol 242: 408-421, 1994.

8. Joshi S and Pringle MJ: ATP synthase complex from bovine heart mitochondria. Passive $\mathrm{H}^{+}$conduction through mitochondrial coupling factor 6-depleted F0 complexes. J Biol Chem 264: 15548-15551, 1989.

9. Zhu H, Chen L, Zhou W, Huang Z, Hu J, Dai S, Wang X, Huang X and He C: Over-expression of the ATP5J gene correlates with cell migration and 5-fluorouracil sensitivity in colorectal cancer. PLoS One 8: e76846, 2013.

10. Yang W, Lu Y, Xu Y, Xu L, Zheng W, Wu Y, Li L and Shen P: Estrogen represses hepatocellular carcinoma (HCC) growth via inhibiting alternative activation of tumor-associated macrophages (TAMs). J Biol Chem 287: 40140-40149, 2012.

11. Bjerregaard H, Pedersen S, Kristensen SR and Marcussen N: Reference genes for gene expression analysis by real-time reverse transcription polymerase chain reaction of renal cell carcinoma. Diagn Mol Pathol 20: 212-217, 2011.

12. Livak KJ and Schmittgen TD: Analysis of relative gene expression data using real-time quantitative PCR and the 2(-Delta Delta C(T)) method. Methods 25: 402-408, 2001.

13. Carson MB and Lu H: Network-based prediction and knowledge mining of disease genes. BMC Med Genomics 8 (Suppl 2): S9, 2015. 
14. Zamani-Ahmadmahmudi M, Najafi A and Nassiri SM: Reconstruction of canine diffuse large B-cell lymphoma gene regulatory network: Detection of functional modules and hub genes. J Comp Pathol 152: 119-130, 2015.

15. Lu P, Weaver VM and Werb Z: The extracellular matrix: A dynamic niche in cancer progression. J Cell Biol 196: 395-406, 2012.

16. Hynes RO: The extracellular matrix: Not just pretty fibrils. Science 326: 1216-1219, 2009.

17. Gilkes DM, Semenza GL and Wirtz D: Hypoxia and the extracellular matrix: Drivers of tumour metastasis. Nat Rev Cancer 14 430-439, 2014.

18. Coussens LM and Werb Z: Inflammation and cancer. Nature 420 860-867, 2002

19. Martini M, De Santis MC, Braccini L, Gulluni F and Hirsch E PI3K/AKT signaling pathway and cancer: An updated review. Ann Med 46: 372-383, 2014.

20. Groner B and von Manstein V: Jak Stat signaling and cancer: Opportunities, benefits and side effects of targeted inhibition. Mol Cell Endocrinol 451: 1-14, 2017.

21. Cheung M and Testa JR: Diverse mechanisms of AKT pathway activation in human malignancy. Curr Cancer Drug Targets 13 234-244, 2013.

22. Agarwal E, Brattain MG and Chowdhury S: Cell survival and metastasis regulation by Akt signaling in colorectal cancer. Cell Signal 25: 1711-1719, 2013.

23. Chau NM and Ashcroft M: Akt2: A role in breast cancer metastasis. Breast Cancer Res 6: 55-57, 2004

24. Rychahou PG, Kang J, Gulhati P, Doan HQ, Chen LA, Xiao SY, Chung DH and Evers BM: Akt2 overexpression plays a critical role in the establishment of colorectal cancer metastasis. Proc Natl Acad Sci USA 105: 20315-20320, 2008.

25. Agarwal E, Robb CM, Smith LM, Brattain MG, Wang J, Black JD and Chowdhury S: Role of Akt2 in regulation of metastasis suppressor 1 expression and colorectal cancer metastasis. Oncogene 36: 3104-3118, 2017.

26. Ding Z, Xu F, Li G, Tang J, Tang Z, Jiang P and Wu H: Knockdown of Akt 2 expression by shRNA inhibits proliferation, enhances apoptosis, and increases chemosensitivity to paclitaxel in human colorectal cancer cells. Cell Biochem Biophys 71: 383-388, 2015.

27. Prensner JR and Chinnaiyan AM: The emergence of lncRNAs in cancer biology. Cancer Discov 1: 391-407, 2011.

28. Li S, Huang Y, Huang Y, Fu Y, Tang D, Kang R, Zhou R and Fan XG: The long non-coding RNA TP73-AS1 modulates HCC cell proliferation through miR-200a-dependent HMGB1/RAGE regulation. J Exp Clin Cancer Res 36: 51, 2017.

29. Zhu G, Wang S, Chen J, Wang Z, Liang X, Wang X, Jiang J, Lang $\mathrm{J}$ and Li L: Long noncoding RNA HAS2-AS1 mediates hypoxia-induced invasiveness of oral squamous cell carcinoma. Mol Carcinog 56: 2210-2222, 2017.
30. Zhang H, Xiong Y, Xia R, Wei C, Shi X and Nie F: The pseudogene-derived long noncoding RNA SFTA1P is downregulated and suppresses cell migration and invasion in lung adenocarcinoma. Tumour Biol 39: 1010428317691418, 2017.

31. Song H, He P, Shao T, Li Y, Li J and Zhang Y: Long non-coding RNA XIST functions as an oncogene in human colorectal cancer by targeting miR-132-3p. J BUON 22: 696-703, 2017.

32. Fang J, Sun CC and Gong C: Long noncoding RNA XIST acts as an oncogene in non-small cell lung cancer by epigenetically repressing KLF2 expression. Biochem Biophys Res Commun 478: 811-817, 2016

33. Yao Y, Ma J, Xue Y, Wang P, Li Z, Liu J, Chen L, Xi Z, Teng H, Wang Z, et al: Knockdown of long non-coding RNA XIST exerts tumor-suppressive functions in human glioblastoma stem cells by up-regulating miR-152. Cancer Lett 359: 75-86, 2015.

34. Wu X, Dinglin X, Wang X, Luo W, Shen Q, Li Y, Gu L, Zhou Q, Zhu H, Li Y, et al: Long noncoding RNA XIST promotes malignancies of esophageal squamous cell carcinoma via regulation of miR-101/EZH2. Oncotarget 8: 76015-76028, 2017.

35. Wang H, Shen Q, Zhang X, Yang C, Cui S, Sun Y, Wang L, Fan X and $\mathrm{Xu}$ S: The long non-coding RNA XIST controls non-small cell lung cancer proliferation and invasion by modulating miR-186-5p. Cell Physiol Biochem 41: 2221-2229, 2017.

36. Kara M, Yumrutas O, Ozcan O, Celik OI, Bozgeyik E, Bozgeyik I and Tasdemir S: Differential expressions of cancer-associated genes and their regulatory miRNAs in colorectal carcinoma. Gene 567: 81-86, 2015.

37. Bione S, Rizzolio F, Sala C, Ricotti R, Goegan M, Manzini MC, Battaglia R, Marozzi A, Vegetti W, Dalprà L, et al: Mutation analysis of two candidate genes for premature ovarian failure, DACH2 and POF1B. Hum Reprod 19: 2759-2766, 2004.

38. Crespi A, Bertoni A, Ferrari I, Padovano V, Della Mina P, Berti E, Villa A and Pietrini G: POF1B localizes to desmosomes and regulates cell adhesion in human intestinal and keratinocyte cell lines. J Invest Dermatol 135: 192-201, 2015.

39. Marzinke MA and Clagett-Dame M: The all-trans retinoic acid (atRA)-regulated gene Calmin (Clmn) regulates cell cycle exit and neurite outgrowth in murine neuroblastoma (Neuro2a) cells. Exp Cell Res 318: 85-93, 2012.

This work is licensed under a Creative Commons Attribution-NonCommercial-NoDerivatives 4.0 International (CC BY-NC-ND 4.0) License. 